\title{
Artificial neural network for determining the hedonic score of texture of and distinguishing different grades of ham sausages
}

\author{
Lintao $\mathrm{ZHU}^{1}$, Wenbiao WU $\mathrm{W}^{1,2 *}$
}

\begin{abstract}
The preference of consumers of ham sausages is mainly determined on its texture. A method of determining hedonic score of texture and distinguishing different grades of ham sausages based on artificial neural network was established in this study. The topological texture of the artificial neural network was developed on the basis of analyzing the hardness, springiness, cohesiveness and adhesiveness measured by a texture analyzer and the hedonic score of texture measured by a sensorial test. The simulation result indicated that the hedonic score of texture predicted by the artificial neural network was well correlated with that obtained by the sensorial test and they were not significantly different from each other. Particularly, cluster analysis proved that the hedonic score of texture predicted by the neural network well discriminated different grades of ham sausages.
\end{abstract}

Keywords: texture profile; sensorial test; BP neural network; gelling foods.

Practical Application: The method established in this study should be applicable in predicting the consumer's acceptability of texture of ham sausages. This method can also distinguish different grades of pork ham sausages so that it can be used for the quality control during the period of production, sale and development of new products.

\section{Introduction}

The yield of ham sausages in China is more than 2 million tons per year (Guo, 2013). It accounts for one third of total annual yield of all the meat products manufactured in China. Chinese National Standard- GB/T 20712-2006 mandatorily requires sensorial test on the texture (particularly including springiness) of ham sausages before delivery. The problem is that large numbers of participants $(\geq 50)$ are needed for obtaining accurate and un-biased results via a sensorial test (Ma et al., 2005) though it still appears to be the major method of evaluating consumer's preference of texture till now (Guo, 2013). This way may not be suitable for routine analysis because it is very costly and therefore difficult to undertake.

A preliminary study carried out in our laboratory indicated that different grades of major ham sausages produced in China contain similar varieties of major components (i.e. meats and starches) so that their taste is not significantly different. However, their texture is very different from each other.

Ham sausages as a kind of gelling food must have brittle and elastic texture which is the preference of their consumers. This is the distinct property of ham sausages which is greatly different from other kinds of sausages or meat products (except for ham meats). These should be the reason for the inclusion of sensorial test on texture in the standard of ham sausages (Wu et al., 2013; Wu, 2013; Rohm, 1990). Furthermore, the need of performing sensory methods based on consumer perception has been reported in the literature (Santos et al., 2015; Horita et al., 2017). Although textural profiles analyzer has been increasingly used for the determination of differences in texture among different samples (Romero de Ávila et al., 2014; Hopkins et al., 2013a,b; Huda et al., 2010), scientific research on the correlation between its measurement and hedonic score obtained by a sensorial test is not adequate because of inadequacy of investigation. An artificial neural network may be useful for predicting hedonic score of ham sausages based on instrumental measurement since its application to the analysis of other foods has been reported to be feasible (Gaze et al., 2015; Cruz et al., 2009).

The aim of this study was to develop an artificial neural network for determining hedonic score of texture and discriminating different grades of ham sausages based on the result obtained by a texture analyzer and a sensorial test for quality control. The texture characteristics involved include hardness $(\mathrm{H})$, springiness $(\mathrm{S})$, cohesiveness (C) and adhesiveness (A). The studying materials include 3 grades of ham sausages which are commercially produced in China.

\section{Materials and methods}

\subsection{Materials}

The samples (300, each weighing $68 \mathrm{~g}$ ) of starch-containing pork ham sausages (ready-to-eat foods) made by Shuanhui Enterprise Group Ltd., Henan, PRC and by Jinluo Enterprise Group Ltd., Xincheng, Linyi, Shandong, PRC were bought from a local market in Beibei, Chongqing, PRC. They included $1 / 3$ extremely superior grade, $1 / 3$ superior grade, and $1 / 3$ regular grade. They were stored at $20-22{ }^{\circ} \mathrm{C}$ for $18 \mathrm{~h}$ prior to sensory evaluation and instrumental measurement at the same temperature range. 


\subsection{Sensorial test on hedonic scores of texture of ham sausages}

Sixty panelists participated in the sensorial test and they tasted a total number of 300 starch-containing pork ham sausages (three grades; one hundreds of each grade). Males or females accounted for $50 \%$, respectively. The panelists included: 10 males and 10 females being 19-25 years old, 10 males and 10 females being 26-35 years old, 10 males and 10 females being $>35$ - 50 years old. The taste of sample of each grade involved 20 panelists. Each panelist tasted 5 samples. All the panelists were asked to immediately complete a form and give the scores of texture after each tasting. The hedonic scores of texture were quantified by using a 9-point hedonic scale ( 1 = dislike extremely, 2 = dislike very much, 3 = dislike moderately, $4=$ dislike slightly, $5=$ neither dislike nor like, $6=$ like slightly, $7=$ like moderately, $8=$ like very much, 9 = like extremely) (Hayes et al., 2014).

Ten cylinders of approximately $5 \mathrm{~mm}$ height were prepared by slicing half of each ham sausage of each grade as sensorial tasting samples for each panelist. One piece of the ham sausage cylinder was served on a plate which was anonymously coded and presented in a random order. Before the test, all panelists were briefed orally and they had written instructions to avoid the effect of aftertastes on the score of texture given by them (carrying out mouthwash 3 times by drinking water between each taste). Ten replicates of the taste for each ham sausage sample of each grade were carried out by each participant. The taste of each ham sausage of each grade included two sessions at $1 \mathrm{~h}$ interval; each session evaluated five replicates of the sample in a randomized order. The correlation between the hedonic score of texture of half a ham sausage sample and the textural properties $(\mathrm{H}, \mathrm{S}, \mathrm{C}$ and $\mathrm{A})$ of another half of the same sample measured by the texture analyzer (see "Texture property analysis of ham sausages") was analyzed.

\subsection{Texture property analysis of ham sausages}

The remaining half of each ham sausage sample after the sensorial test described in the above section was employed in measuring $\mathrm{H}$ (the maximum peak force of first compression to deform the sample), $\mathrm{S}$ (the distance that the sample recovered its height between the first and second compressions), $\mathrm{C}$ (the positive ratio of the second compression area to the first compression area) and A (the energy $(\mathrm{mJ})$ required to separate a probe from the sample on the return stroke). The texture profile of the ham sausage sample (cut into a length of $20 \mathrm{~mm}$, and $17 \mathrm{~mm}$ diameter pieces) which was orientated towards the axis of the testing probe was measured by using a texture analyzer (CT3 manufactured by Brookfield Engineering Laboratories, INC., USA). The test was carried out at $22{ }^{\circ} \mathrm{C}$ by using the following settings: pre-test speed, $2.0 \mathrm{~mm} / \mathrm{s}$; test speed, $1.0 \mathrm{~mm} / \mathrm{s}$; post test speed, $1.0 \mathrm{~mm} / \mathrm{s}$; compression ratio, $75 \%$ of its depth (Mittal et al., 1992) for measuring the $\mathrm{H}$ of all samples, $60 \%$ of its depth for measuring the S, C and A of extremely superior and superior grade samples, $40 \%$ of its depth for measuring the $\mathrm{S}, \mathrm{C}$ and $\mathrm{A}$ of regular grade samples; time interval between the first and second compressions, $5 \mathrm{~s}$; testing probe, TA25 (50 $\mathrm{mm}$ diameter). Three replicates of measurement for each sample were carried out.

\subsection{Establishment of BP neural network}

Matlab (R2015b) developed by MathWorks, USA was used to establish BP (back propagation) neural network. The simulation of prediction model was constantly debugged. The maximum training times were 10000 . The convergence error was 0.01 . The learning rate was 0.05 . The momentum constant was 0.9. Mean-square error (MSE) was used as the most important index for prediction accuracy of the neural network model. $\mathrm{R}$ validation set and $\mathrm{R}$ testing set were used as reference indexes. All these practices together were supposed to be able to find the most suitable prediction model. Finally, the TRAINGDX (i.e. $m c^{\star} \mathrm{dXprev}+\mathrm{lr}{ }^{\star} \mathrm{mc}{ }^{*} \mathrm{dpref} / \mathrm{dX}$; mc being 0.9 which was momentum parameter, lr being 0.01 which was the learning rate, $\mathrm{dXprev}$ being the previous weight and deviation change) algorithm was invoked to establish the network.

The neural network consists of one input layer, one hidden layer, one output layer and the connection of neurons. In this study, the input layer included 4 different textural characteristics (i.e. $x_{1}-H, x_{2}-S, x_{3}-C$ and $x_{4}-A$ ). The output layer was the hedonic score of texture of ham sausages. The 300 pair data of characteristics ( H, S, C and A) and hedonic scores of texture of ham sausages collected were randomly divided into 3 groups: training set (80\%), validation set (10\%) and testing set (10\%), which were proper numbers of data which can be used with a total of 300 samples in these steps.

For solving the problem of inconsistence of input variables and their numbers, transformation function (premnmx; S1) was used to normalize them at the interval of $[-1,+1]$.

The transfer function from the input layer to the hidden layer was tansig (S2 - Supplementary Material). The function from the hidden layer to the output layer was purelin (S3 - Supplementary Material). The output values were normalized by postmnmx (S4 - Supplementary Material).

\subsection{Analysis of the composition of the ham sausage samples}

The content of water, protein, total saccharide, fat, and sodium chloride in the ham sausage samples was determined according to China National Standards (2003, 2008a,b, 2010a,b, 2016) GB 5009.3-2010, GB 50095-2010, GB/T 9695.31-2008, GB/T 5009.6-2003, and GB/T 12457-2008, respectively. For water determination, the sample was directly dried to a constant weight at $101.3 \mathrm{kPa}$ at $101-105^{\circ} \mathrm{C}$ and the water content was calculated according to the weight before and after drying. For protein determination, Kjeldahl procedure was used (Chinese National Standard GB 50095-2010). For total saccharide including starches determination, the sample was extracted by hot water and the extract was treated by phenol ( $5 \mathrm{~g}$ in $100 \mathrm{~mL}$ water) and sulphuric acid $(1.84 \mathrm{~g} / \mathrm{mL})$ solution after the starches were hydrolyzed by amylase followed by measuring the absorbance of colored solution. The content of starch was measured according to Chinese National Standard GB/T 5009.9-2016. For fat determination, Soxhlet method was used while sodium chloride was determined by indirect precipitation titration (Volhard) (Chinese National Standard GB/T 5009.6-2003). 


\subsection{Statistics analysis}

The correlation analysis was conducted by using IBM SPSS Statistics 19 while K-means cluster analysis was carried out by MatlabR2015b. A student's t test was used to test the difference in paired data. All the data including H, S, C, A and HS were entered into the SPSS data editor. F test was used to test the variance of data of each grade ham sausages measured by texture analyzer and by sensorial test. Then all coefficients were calculated by multifactor linear regression with $\mathrm{HS}$ as the dependent variable and $\mathrm{H}, \mathrm{S}, \mathrm{C}$ and $\mathrm{A}$ as variables.

\section{Results and discussion}

\subsection{Characteristics and hedonic scores of texture of ham sausages}

The characteristics of texture which were measured by texture analyzer and the hedonic scores of texture which were estimated by sensorial test are shown in Table 1 . The hedonic scores obtained were believed to be accurate since the sensory test using 9 point scale which was employed in this study has been widely applied in the area of food science and technology (Vidal et al., 2019). These data were used for the establishment of the neural network.

\subsection{Establishment of neural network and the prediction of hedonic score}

Selection of nodes in hidden layer. The nodes in hidden layer from 5 to 30 were tested. The errors of predicting hedonic scores by the neural network including different nodes in the hidden layer with the interval of five nodes were shown in Figure 1. It can be seen from this figure that 15 nodes in the hidden layer gave the lowest prediction error which was only 0.0098160 among all tested. The further test covered 15 - 20 nodes with the interval of one node indicated that 16 nodes in the hidden layer gave the lowest prediction error which was only 0.0069791 among all tested. When 16 nodes in the hidden layer were selected, the MSE of value of hedonic score of texture predicted by the neural network was 0.043 , which was much lower than 0.05 .

Established neural network. The finally established neural network shown in Figure 2 included 1 input layer (having 4 input variables), 1 hidden layer (having 16 nodes) and 1 output layer. By using this neural network, the input of $\mathrm{H}, \mathrm{S}, \mathrm{C}$ and $\mathrm{A}$ (the input layer) gave a hedonic score (the output layer). Data from 30 samples in the validation set were used to validate this neural network. The scatter diagram obtained by plotting values for hedonic score of texture measured by sensorial test against that predicted by the neural network and the difference between these two sets of data are shown in Figure 3. Sample 14 had the

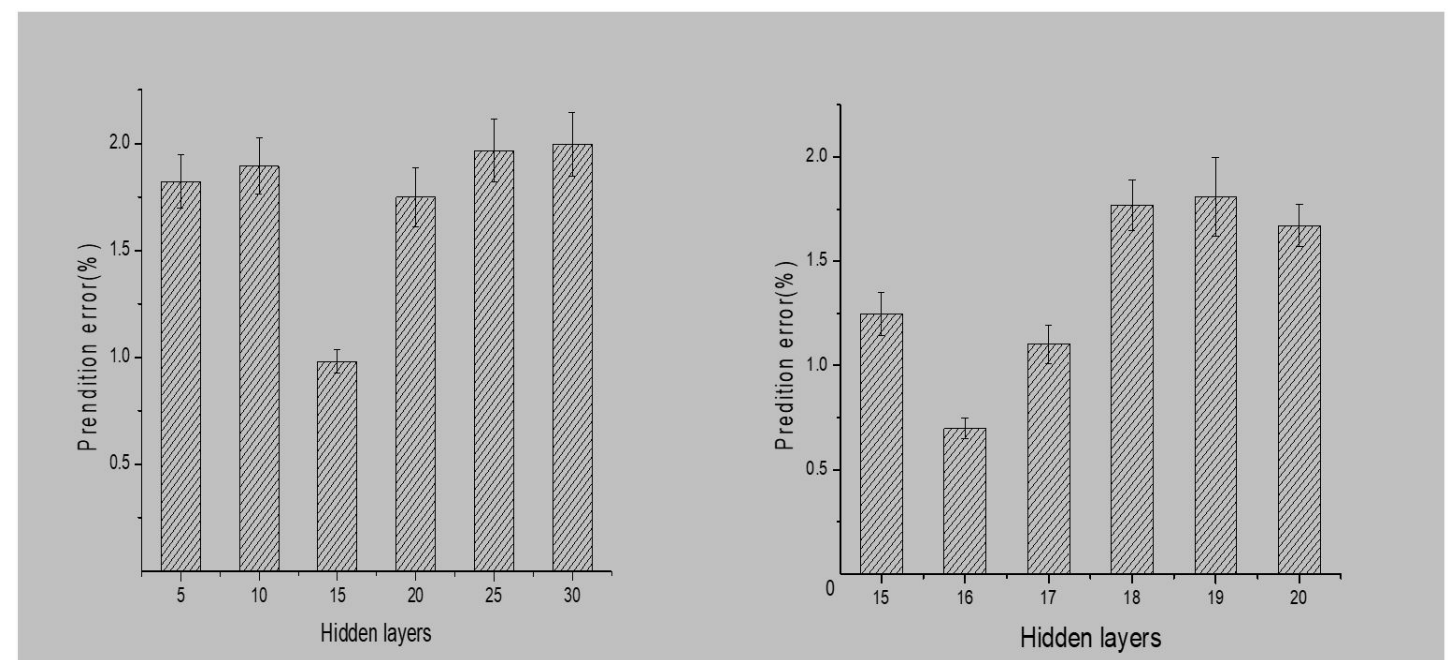

Figure 1. Effect of different numbers of nodes in the hidden layer on the error of hedonic score of texture of ham sausages predicted by the neural network.

Table 1. Textural characteristic of ham sausages measured by a texture analyzer and hedonic score of their texture estimated by a sensorial test for different sets.

\begin{tabular}{|c|c|c|c|c|c|c|}
\hline Sets & Grade & Hardness (g) & Springiness $(\mathrm{mm})$ & Cohesiveness & Adhesiveness (mJ) & Hedonic score \\
\hline \multirow{3}{*}{$\begin{array}{l}\text { Training (mean } \pm \text { SE; } \\
\mathrm{n}=80)\end{array}$} & Regular & $2254 \pm 143.98$ & $5.91 \pm 0.49$ & $0.32 \pm 0.03$ & $360.06 \pm 14.48$ & $4.75 \pm 0.15$ \\
\hline & Superior & $3507.99 \pm 225.75$ & $9.36 \pm 0.34$ & $0.21 \pm 0.03$ & $526.00 \pm 57.8$ & $6.82 \pm 0.22$ \\
\hline & Extremely superior & $4295.45 \pm 289.80$ & $9.83 \pm 0.36$ & $0.17 \pm 0.02$ & $855.41 \pm 54.04$ & $8.64 \pm 0.23$ \\
\hline \multirow{3}{*}{$\begin{array}{l}\text { Validation (mean } \pm \text { SE; } \\
\mathrm{n}=10)\end{array}$} & Regular & $2212.90 \pm 171.21$ & $5.76 \pm 0.48$ & $0.31 \pm 0.02$ & $362.90 \pm 22.56$ & $4.71 \pm 0.17$ \\
\hline & Superior & $3531.60 \pm 290.06$ & $9.38 \pm 0.29$ & $0.22 \pm 0.03$ & $550.20 \pm 45.1$ & $6.89 \pm 0.22$ \\
\hline & Extremely superior & $4299.20 \pm 187.82$ & $9.78 \pm 0.27$ & $0.17 \pm 0.02$ & $864.20 \pm 41.61$ & $8.43 \pm 0.21$ \\
\hline \multirow{3}{*}{$\begin{array}{l}\text { Testing (mean } \pm \text { SE; } \\
\mathrm{n}=10 \text { ) }\end{array}$} & Regular & $2282.71 \pm 151.31$ & $5.65 \pm 0.51$ & $0.32 \pm 0.01$ & $365.90 \pm 21.47$ & $4.67 \pm 0.16$ \\
\hline & Superior & $3542.59 \pm 289.12$ & $9.34 \pm 0.25$ & $0.21 \pm 0.02$ & $554.80 \pm 44.75$ & $6.71 \pm 0.21$ \\
\hline & Extremely superior & $4308.20 \pm 175.95$ & $9.86 \pm 0.26$ & $0.18 \pm 0.02$ & $873.10 \pm 39.5$ & $8.61 \pm 0.24$ \\
\hline
\end{tabular}

${ }^{\star} \mathrm{F}$ test indicated that the variance of all data of each grade was not statistically significant $(\mathrm{P}>0.05)$. 


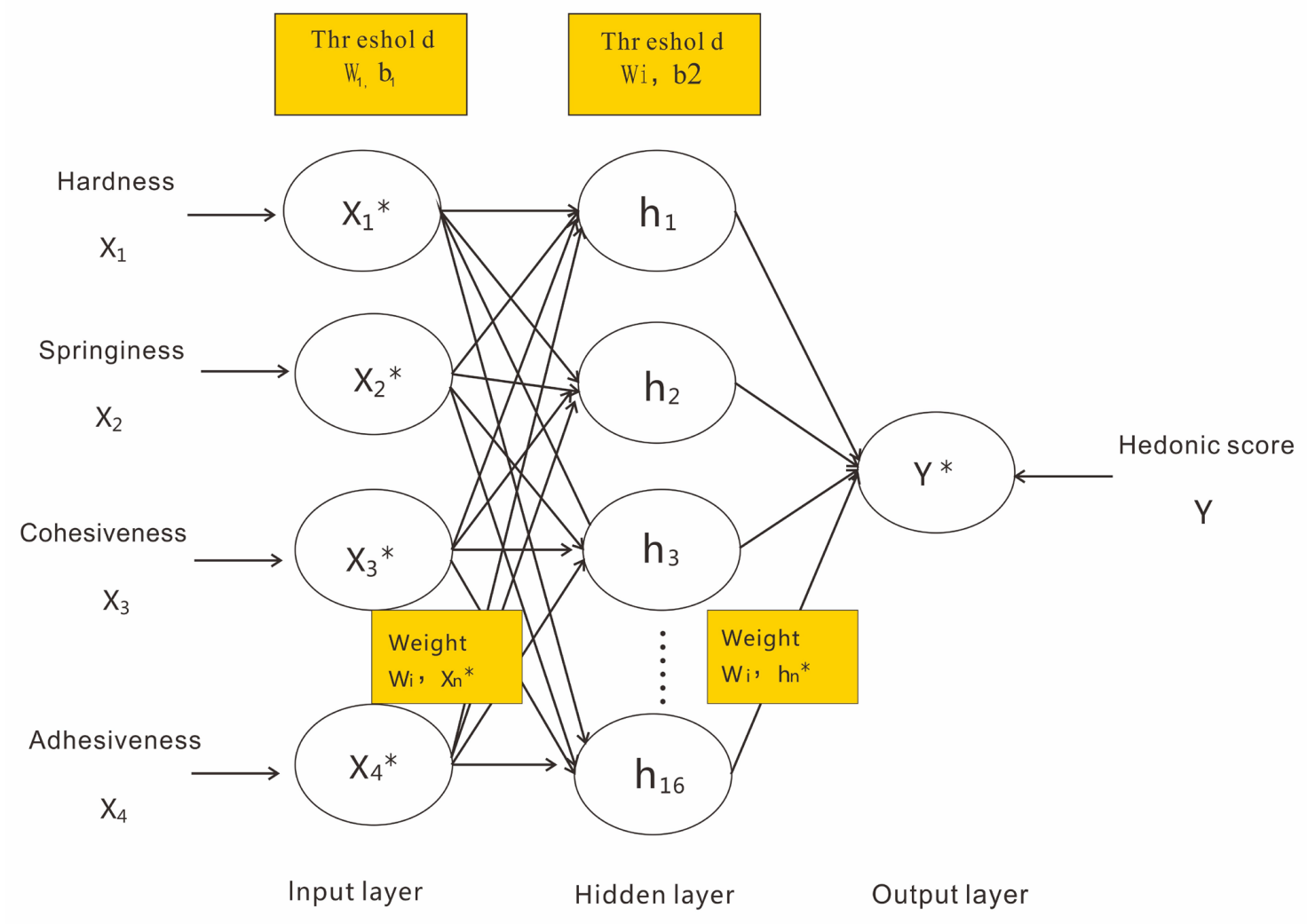

Figure 2. Neural network for predicting hedonic score of texture of ham sausages based on the measurement of their hardness, springiness, cohesiveness and adhesiveness.
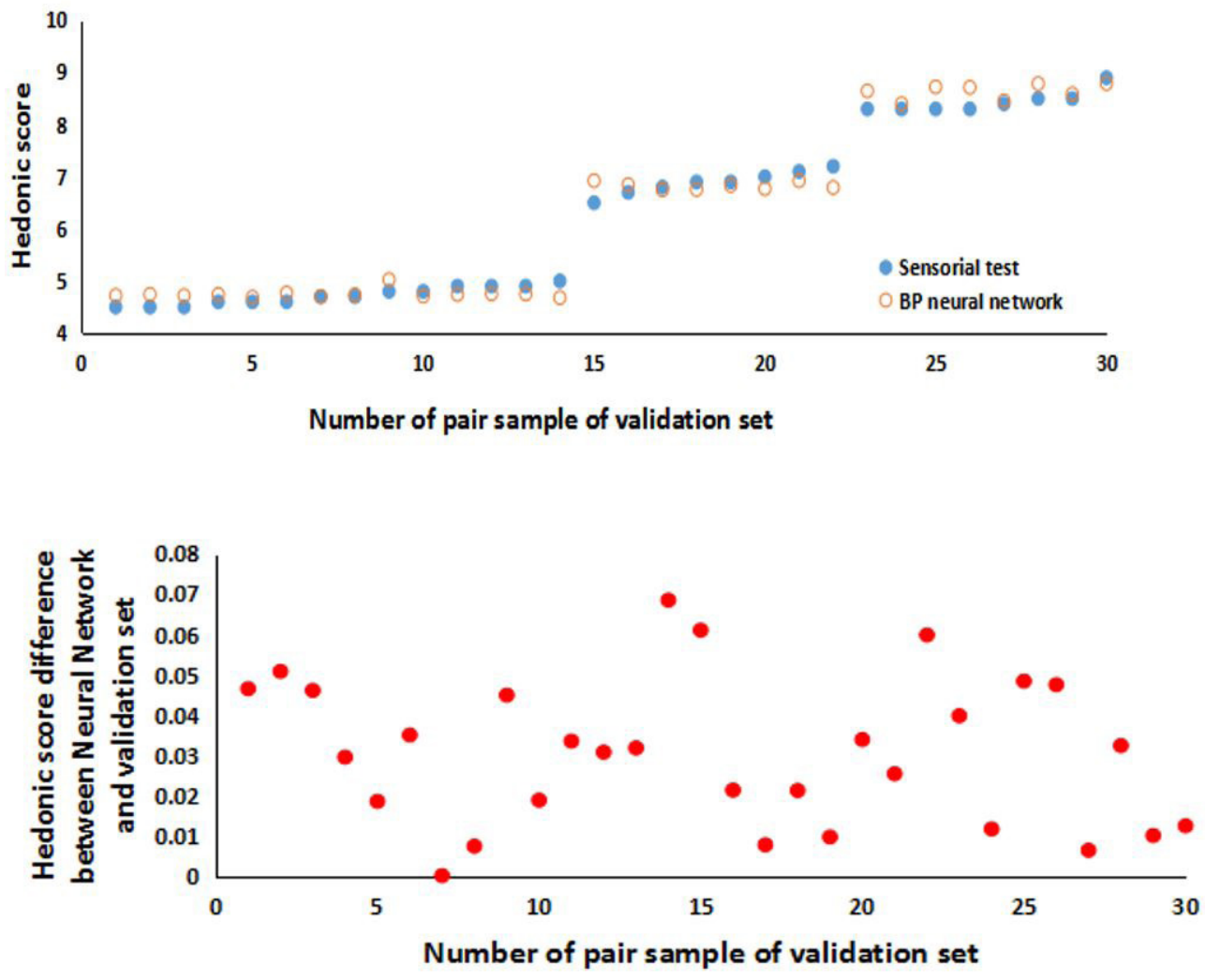

Figure 3. Comparison of hedonic score obtained by sensorial test and that predicted by the neural network as well as the difference between these two sets of data in validation set. The difference is in the absolute values. 
biggest difference (0.068) between the hedonic score of texture obtained by the sensorial test and that predicted by the neural network among all samples investigated. However, the difference was not statistically significant $(\mathrm{P}>0.05)$. The correlation coefficient between the values of hedonic score of texture estimated by sensorial test and that obtained from the validation set was $0.991(\mathrm{P}(\mathrm{sig})=0.00<0.01)$. The equation which well indicated the linear correlation between the results of sensorial test and the validation set was as the following:

$y=0.964 x+0.177\left(R^{2}=0.982\right)$

In the equation, $y$ represents the hedonic score predicted by the neural network while $\mathrm{x}$ is that obtained by sensorial test. Therefore, the hedonic scores of texture measured by sensorial test and that predicted by the neural network have good linear relationship.

Further evaluation of reliability of the neural network for predicting hedonic score. For further testing the reliability of the neural network for predicting hedonic score of texture of ham sausages established in this study, data from 30 samples in the testing set were analyzed. The original data, i.e. the average hedonic score of texture of ham sausages calculated from the value obtained by sensorial test and that predicted by the neural network established in this study based on the measured value of H, S, C and A shown in Table 1, which were not significantly different $(\mathrm{P}>0.05)$.

The scatter diagram obtained by plotting values for hedonic score of texture measured by sensorial test against that predicted by the neural network and the difference between these two sets of data are shown in Figure 4. Sample 2 had the biggest difference (0.073) between the hedonic score of texture obtained by the sensorial test and that predicted by the neural network among all samples investigated. However, the difference was not statistically significant $(\mathrm{P}>0.05)$.

The correlation coefficient between the values of hedonic score of texture estimated by sensorial test and that predicted by the neural network was $0.993(\mathrm{P}(\mathrm{sig})=0.00<0.01$; $\mathrm{RMSE}=0.0676$; $\mathrm{RSD}=0.18617$ ). Therefore, this test again proved that the hedonic scores of texture measured by sensorial test and that predicted by the neural network had good linear relationship.

\subsection{Comparison of the neural network with multiple regression}

For comparison, a multiple regression model was established by using the same data of the training set for establishing the neural network, which was as the following:
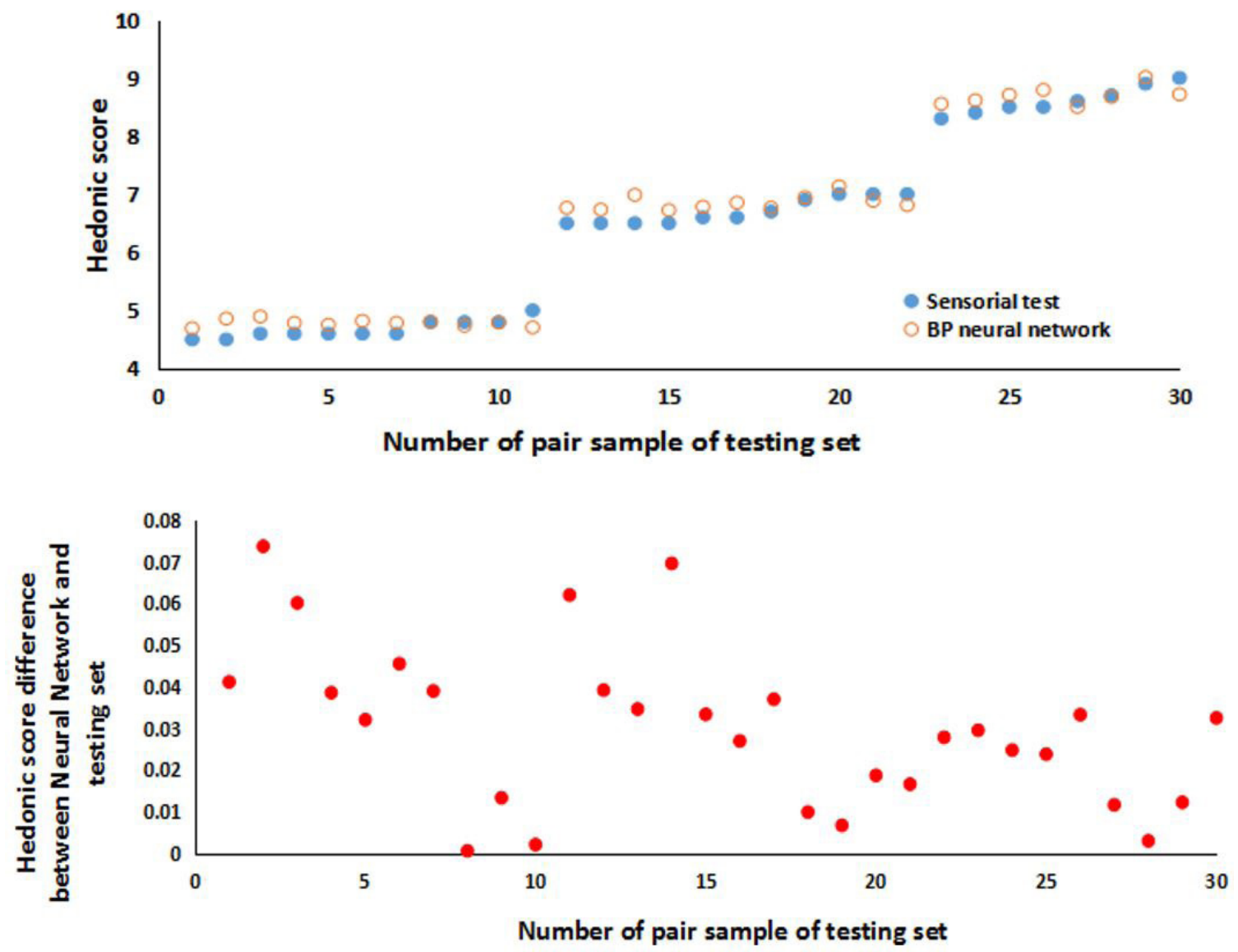

Figure 4. Comparison of hedonic scores obtained by sensorial test and that predicted by the neural network as well as the difference between these two sets of data in testing set. The difference is in the absolute values. 


$$
y=1.811+0.578 x_{1}+0.184 x_{2}-1.954 x_{3}+0.003 x_{4}\left(R^{2}=0.867\right)
$$

In the equation, $\mathrm{y}$ represents the hedonic score of texture predicted while $\mathrm{x}_{1}, \mathrm{x}_{2}, \mathrm{x}_{3}$ and $\mathrm{x}_{4}$ are $\mathrm{H}(\mathrm{kg}), \mathrm{S}(\mathrm{mm}), \mathrm{C}$ and $\mathrm{A}(\mathrm{mJ})$, respectively.

The hedonic score of texture predicted by this multiple regression model (equation (2)) and the neural network, that obtained by sensorial test and the difference between these three methods are indicated in Figure 5. The biggest difference (0.12) between the hedonic score of texture obtained by the sensorial test and that predicted by the multiple regression model was 0.12 (sample 1). The hedonic scores of texture of sample 1, 2, 3, 4, 5, 6, 7, 9 and 10 were significantly different between sensorial test and multiple regression method $(\mathrm{P}<0.05)$. On the other hand, there was no difference in the hedonic scores of texture of all samples analyzed between the sensorial test and the neural network.

\subsection{Correlation between the composition of ham sausages and their hedonic score predicted by the neural network or texture}

Table 2 indicates that the main components of ham sausages had a linear relationship with their hedonic score predicted by the neural network or some textural properties measured by the texture analyzer. The protein content positively correlated with the hedonic score predicted by the neural network and the textural properties including hardness, springiness and adhesiveness. The starch or total sugar content negatively correlated with the hedonic score predicted by the neural network and the textural properties including hardness, springiness and adhesiveness. These results also indicate that the neural network established in this study can be applied to the evaluation of nutritional quality of ham sausages.

\subsection{Application of the neural network}

The neural network established by using a texture analyzer should have wide application in the production of ham sausages and development of this kind of new product for quality control. It can be used to distinguish different grades of ham sausages (Figure 6). Particularly, this study found that the hedonic score of texture predicted by the neural network were positively or negatively correlated with the content of proteins $(14.00 \pm 0.44 \%$ in extremely superior grade, $12.10 \pm 0.32 \%$ in superior grade and $11.00 \pm 0.36 \%$ in regular grade) or starches $(6.50 \pm 0.24 \%$ in extremely superior grade, $8.10 \pm 0.22 \%$ in superior grade and $10.10 \pm 0.31 \%$ in regular grade), respectively. Therefore, the method of predicting hedonic score developed in this study can be applied in the quality control of starch-containing pork ham
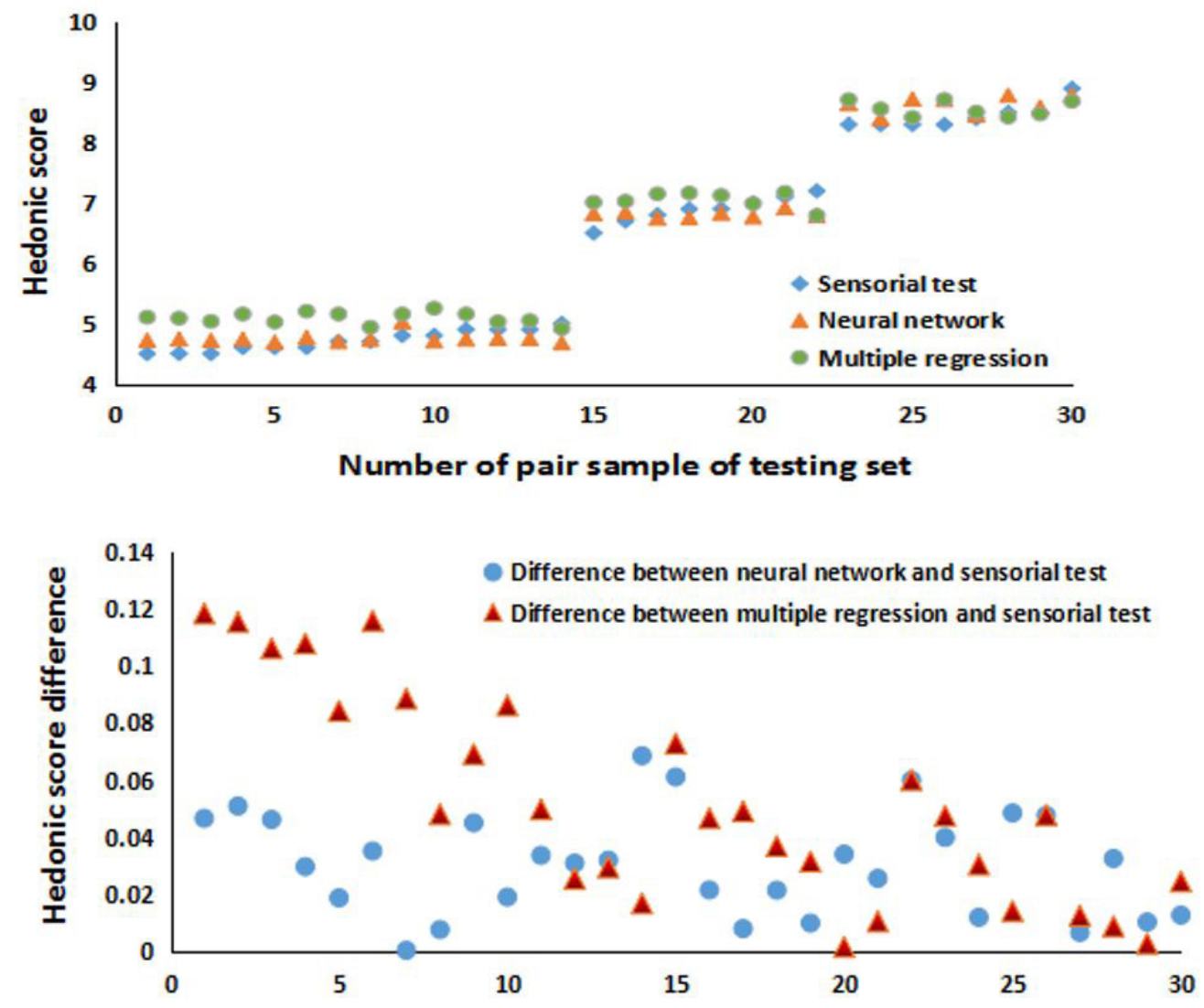

Number of pair samples of testing set

Figure 5. Comparison of hedonic scores obtained by sensorial test and that predicted by the neural network or by multiple regression and the difference between these three sets of data in testing set. The difference is in the absolute values. 
Table 2. Correlation between composition (\%) of 3 groups of ham sausages and their hedonic score predicted by neural network or textural properties $\left(\mathrm{x} \pm \delta_{\mathrm{n}-1} ; \mathrm{n}=300\right)$.

\begin{tabular}{lcrr}
\hline \multicolumn{1}{c}{ Variety of the ham sausage } & Extremely superior & \multicolumn{1}{c}{ Superior } & Regular \\
\hline Water & $66.02 \pm 0.39$ & $65.40 \pm 0.43$ & $64.80 \pm 0.45$ \\
Protein & $14.00 \pm 0.44$ & $12.10 \pm 0.32$ & $11.00 \pm 0.36$ \\
Saccharide & $6.50 \pm 0.24$ & $8.10 \pm 0.22$ & 7.98 \\
Starch & 6.01 & $13.40 \pm 0.28$ & $10.10 \pm 0.31$ \\
Fat & $12.50 \pm 0.43$ & $1.00 \pm 0.22$ & $13.00 \pm 0.35$ \\
Sodium chloride & $0.98 \pm 0.39$ & $6.81 \pm 0.23$ & $1.10 \pm 0.24$ \\
Hedonic Score & $8.59 \pm 0.24$ & $3527.39 \pm 0.24$ & $4.74 \pm 0.16$ \\
Hardness (g) & $4300.95 \pm 0.26$ & $9.36 \pm 0.51$ & $2250.09 \pm 0.17$ \\
Springiness (mm) & $9.82 \pm 0.68$ & $0.21 \pm 0.04$ & $5.77 \pm 0.21$ \\
Cohesiveness & $0.19 \pm 0.05$ & $543.67 \pm 18.16$ & $0.32 \pm 0.02$ \\
Adhesiveness (m) & $864.24 \pm 18.05$ & & $362.95 \pm 14.61$ \\
\hline
\end{tabular}

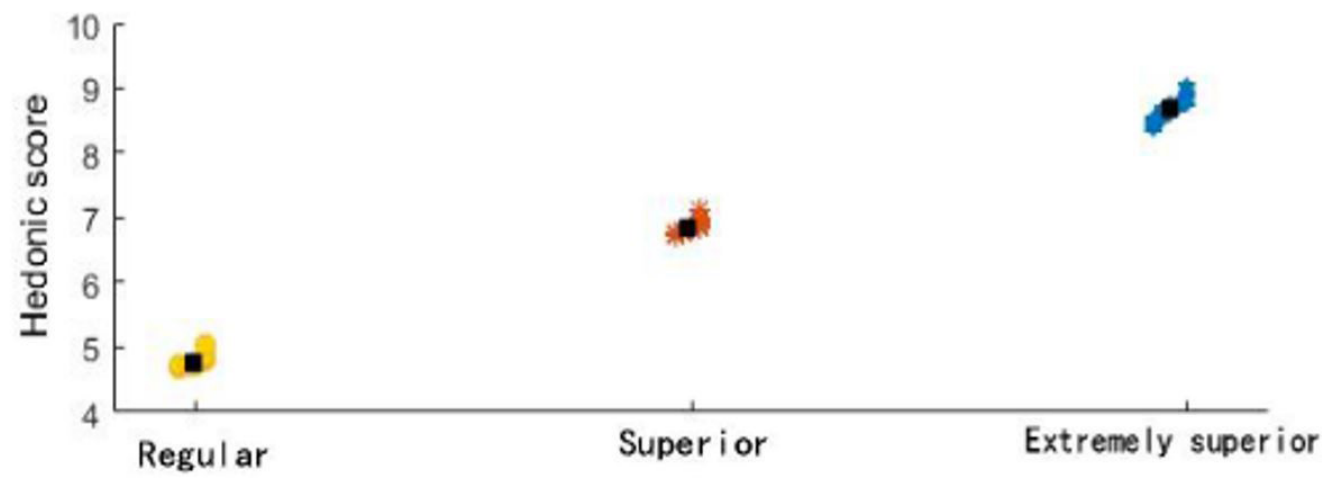

Figure 6. Cluster analysis shown that the hedonic scores predicted by the neural network well distinguish three grades of starch-containing ham sausages.

sausages. It may be readily adapted for predicting the hedonic score of texture of ham meat or hotdog.

The procedure of establishing the neural network can be applied to the prediction of structural hedonic score of other foods, particularly, those with their palatability greatly determined on their texture. For example, it can be applied in the quality control of producing biscuits or other baking products, starchy gelling foods and fruit jelly or the estimation of their consumer acceptability.

A neural network established by using $\mathrm{H}, \mathrm{S}, \mathrm{C}$ and A measured by a texture analyzer should also be appropriate for predicting the grade of any meat or its product. The measurement of these structural properties may also be applied to establish a neural network for predicting the quality of native meat since its application to monitoring the authenticity of milk products was reported to be feasible (Cruz et al., 2009). It should be worthwhile conducting study to compare this method with ultrasound image for evaluating cattle meat (Jang et al., 2015).

\section{Conclusion}

The neural network established by using the data of measured hardness, springiness, cohesiveness and adhesiveness via a texture analyzer in this study can accurately predict the hedonic score of texture of ham sausages. The neural network gave more accurate prediction of hedonic score with respect to the texture of ham sausages as compared with multiple regression method. Furthermore, the hedonic score of texture predicted by the neural network can well distinguish three grades of ham sausages.

\section{References}

China National Standards. (2003, August 11). Determination of fat in foods (GB/T 5009.6-2003). Beijing: General Administration of Quality Supervision, Inspection and Quarantine and Standardization Administration of the People's Republic of China.

China National Standards. (2008a, August 25). Determination of sodium chloride in foods (GB/T 12457-2008). Beijing: General Administration of Quality Supervision, Inspection and Quarantine and Standardization Administration of the People's Republic of China.

China National Standards. (2008b, August 28). Determination of total sugar content in meat products (GB/T 9695.31-2008). Beijing: General Administration of Quality Supervision, Inspection and Quarantine and Standardization Administration of the People's Republic of China.

China National Standards. (2010a, March 26). Determination of protein in foods (GB 5009.5-2010). Beijing: General Administration of Quality Supervision, Inspection and Quarantine and Standardization Administration of the People's Republic of China.

China National Standards. (2010b, March 26). Determination of water in foods (GB 5009.3-2010). Beijing: General Administration of Quality Supervision, Inspection and Quarantine and Standardization Administration of the People's Republic of China. 
China National Standards. (2016, December 23). Determination of starch in foods (GB/T 5009.9-2016). Beijing: General Administration of Quality Supervision, Inspection and Quarantine and Standardization Administration of the People's Republic of China.

Cruz, A. G., Walter, E. H. M., Cadena, R. S., Faria, J. A. F., Bolini, H. M. A., \& Fileti, A. M. F. F. (2009). Monitoring the authenticity of low-fat yogurts by an artificial neural network. Journal of Dairy Science, 92(10), 4797-4804. http://dx.doi.org/10.3168/jds.20092227. PMid:19762794.

Gaze, L. V., Oliveira, B. R., Ferrao, L. L., Granato, D., Cavalcanti, R. N., Conte Júnior, C. A., Cruz, A. G., \& Freitas, M. Q. (2015). Preference mapping of dulce de leche commercialized in Brazilian markets. Journal of Dairy Science, 98(3), 1443-1454. http://dx.doi.org/10.3168/ jds.2014-8470. PMid:25557891.

Guo, X. (2013). The innovation of brand of ham sausage needs perseverance. Farm Products Processing, 12, 64-65.

Hayes, J. E., Raines, C. R., De Pasquale, D. A., \& Cutter, C. N. (2014). Consumer acceptability of high hydrostatic pressure (HHP)-treated ground beef patties. Lebensmittel-Wissenschaft + Technologie, 56(1), 207210. http://dx.doi.org/10.1016/j.lwt.2013.11.014.

Hopkins, D. L., Allingham, P. G., Colgrave, M., \& van de Ven, R. J. (2013a). Interrelationships between measures of collagen, compression, shear force and tenderness. Meat Science, 95(2), 219-223. http://dx.doi. org/10.1016/j.meatsci.2013.04.054. PMid:23747614.

Hopkins, D. L., Lamb, T. A., Kerr, M. J., \& van de Ven, R. (2013b). The interrelationship between sensory tenderness and shear force measured by the G2 Tenderometer and a Lloyd Texture analyser fitted with a Warner-Bratzler head. Meat Science, 93(4), 838-842. http://dx.doi.org/10.1016/j.meatsci.2012.11.052. PMid:23305834.

Horita, C. N., Esmerino, E. A., Vidal, V. A. S., Farah, J. S., Amaral, G. V., Bolini, H. M. A., Cruz, A. G., \& Pollonio, M. A. R. (2017). Sensory profiling of low sodium frankfurter containing garlic products: Adequacy of Polarized Projective Mapping compared with trained panel. Meat Science, 131, 90-98. http://dx.doi.org/10.1016/j. meatsci.2017.05.002. PMid:28500963.

Huda, N., Lin, O. J., Ping, Y. C., \& Nurkhoeriy, T. (2010). Effect of chicken and duck meat ratio on the properties of sausage. International Journal of Poultry Science, 9(6), 550-555. http://dx.doi.org/10.3923/ ijps.2010.550.555.
Jang, E., Cho, H., Kim, E. K., \& Kim, S. (2015, November 18-20). Grade prediction of meat quality in Korean native cattle using Neural Network. In International Conference on Fuzzy Theory and Its Applications - iFUZZY (pp. 18-20). Taiwan: IEEE. http://dx.doi. org/10.1109/iFUZZY.2015.7391889.

Ma, Y., Han, C., \& Liu, J. (2005). Sensory evaluation of food. Beijing: Chemical Industry Press.

Mittal, G. S., Nadulski, R., Barbut, S., \& Negi, S. C. (1992). Textural profile analysis test conditions for meat products. Food Research International, 25(6), 411-417. http://dx.doi.org/10.1016/09639969(92)90165-2.

Rohm, H. (1990). Consumer awareness of food texture in Austria. Journal of Texture Studies, 21(3), 363374. http://dx.doi. org/10.1111/j.1745-4603.1990.tb00485.x.

Romero de Ávila, M. D., Isabel Cambero, M., Ordóñez, J. A., De La Hoz, L., \& Herrero, A. M. (2014). Rheological behaviour of commercial cooked meat products evaluated by tensile test and texture profile analysis (TPA). Meat Science, 98(2), 310-315. http:// dx.doi.org/10.1016/j.meatsci.2014.05.003. PMid:24880977.

Santos, B. A., Campagnol, P. C. B., Cruz, A. G., Galvão, M. T. E. L., Monteiro, R. A., Wagner, R., \& Pollonio, M. A. R. (2015). Check all that apply and free listing to describe the sensory characteristics of low sodium dry fermented sausages: Comparison with trained panel. Food Research International, 76(3), 725-734. http://dx.doi. org/10.1016/j.foodres.2015.06.035. PMid:28455058.

Vidal, V. A. S., Biachi, J. P., Paglarini, C. S., Pinton, M. B., Campagnol, P. C. B., Esmerino, E. A., Cruz, A. G., Morgano, M. A., \& Pollonio, M. A. R. (2019). Reducing 50\% sodium chloride in healthier jerked beef: An efficient design to ensure suitable stability, technological and sensory properties. Meat Science, 152, 49-57. http://dx.doi. org/10.1016/j.meatsci.2019.02.005. PMid:30802818.

$\mathrm{Wu}, \mathrm{W}$. (2013). Basic guidelines for establishing food standards. In C. M. Gagne, \& D. B. Jones (Eds.), Processed foods: quality, safety characteristics and health implications. New York: Nova Science Publishers.

Wu, W., Huang, W., \& Yang, Y. (2013). Principles of quality analysis and critical control points applicable to food processing. In C. M. Gagne \& D. B. Jones (Eds.), Processed foods: quality, safety characteristics and health implications. New York: Nova Science Publishers. 


\section{Supplementary Material}

Supplementary material accompanies this paper.

S2 Transfer function (tansig) from the input layer to the hidden layer

S1 Transformation function (premnmx) for normolizing input variables

S3 Function (purelin) from the hidden layer to the output layer

S4 Function (postmnmx) for normolizing output value

This material is available as part of the online article from http://www.scielo.br/cta 